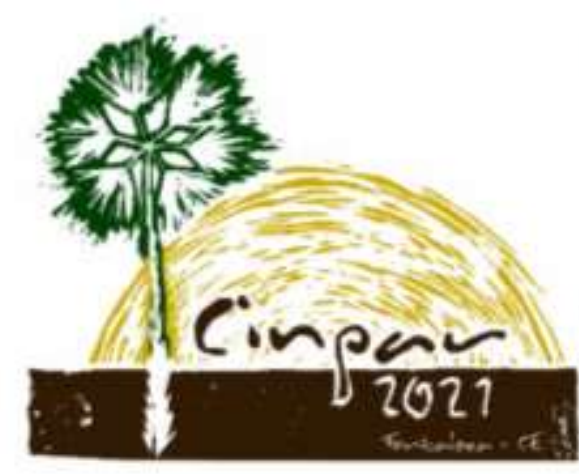

XVII Congresso Internacional sobre Patologia e Reabilitação das Construções

XVII Congreso Internacional sobre Patologia y Rehabilitación de las Construcciones

XVII International Conference on Pathology and Constructions Rehabilitation

FORTALEZA (Brasil), 3 a 5 de junho de 2021

https://doi.org/10.4322/CINPAR.2021.069

\title{
Análise da resistência à compressão axial do concreto a partir de ensaios de esclerometria
}

\section{Analysis of the axial compression strength of concrete from Standard Test Method for Rebound Number of Hardened Concrete} \author{
BORTONE ${ }^{4}$, Rachel Jardim MARTINI ${ }^{5}$, Marcos de Paulo RAMOS ${ }^{6}$ \\ ${ }^{1}$ CEFET-MG, Curvelo, Brasil, laurakaddyjean@ymail.com \\ ${ }^{2}$ CEFET-MG, Curvelo, Brasil, anapaulapaisgott@hotmail.com \\ ${ }^{3}$ CEFET-MG, Curvelo, Brasil, gabrielaandrade74@hotmail.com \\ ${ }^{4}$ CEFET-MG, Curvelo, Brasil, thiago.bortone@cefetmg.br \\ ${ }^{5}$ CEFET-MG, Curvelo, Brasil, martini@cefetmg.br \\ ${ }^{6}$ CEFET-MG, Curvelo, Brasil, marcos.ramos@cefetmg.br
}

Laura Kaddyjean Oliveira SOARES ${ }^{1}$, Ana Paula Pais GOTT², Gabriela Santos DE ANDRADE ${ }^{3}$, Thiago Pena

\begin{abstract}
Resumo: Os ensaios não destrutivos são ferramentas de grande importância quanto à determinação das características das estruturas de concreto, podendo ser usada durante sua fase inicial ou em estruturas existentes que necessitem de avaliação. A investigação tradicional das propriedades mecânicas das estruturas inicia-se com a retirada de corpos de prova a fim de realizar ensaios de resistência à compressão axial do concreto, de acordo com as normas vigentes. Esta propriedade mecânica é um dos parâmetros fundamentais no dimensionamento de estruturas de concreto armado, e comprova que o concreto utilizado atende às exigências estabelecidas em projetos. Entretanto esse método causa danos que podem comprometer a estrutura, além de utilizar equipamentos e mão de obra especializada para a retirada de testemunhos, o que deixa o processo mais oneroso. É válido destacar a necessidade de estudos e verificação de viabilidade por outros métodos de avaliação, quanto à resistência do material "in loco", em diferentes idades, sem que ocorra danos nas peças estruturais a serem analisadas. Este trabalho aborda a análise da evolução da resistência à compressão axial do concreto utilizando o esclerômetro, método de ensaio não destrutivo amplamente utilizado que causa pouco ou nenhum dano à estrutura. Os corpos de provas foram confeccionados no Município de Curvelo/MG, nas mesmas condições de traço, cura, umidade e temperatura. Após a sua desenforma foi transportado, em cura úmida, para o laboratório de ensaios Consultare Labcon em Belo Horizonte/MG, onde realizou-se os ensaios de resistência à compressão e índice esclerométrico em diferentes idades. A correlação da resistência à compressão axial do concreto com o valor do índice esclerométrico, foi feita gerando uma curva de calibração para o concreto estudado. Obteve-se um bom índice de correlação $\left(R^{2}=0,95\right)$ para as idades analisadas.
\end{abstract}

Palavras-chave: Ensaios não destrutivos; Esclerômetro de reflexão; Resistência à compressão.

Abstract: Non-destructive tests are tools of great importance in determining the characteristics of concrete structures, and can be used during their initial phase or in existing structures that require evaluation. The traditional investigation of the mechanical properties of the structures begins with the removal of specimens in order to carry out tests of resistance to axial compression of the concrete, according to the current regulations. This mechanical property is one of the fundamental parameters in the design of reinforced concrete structures, and proves that the concrete used meets the requirements established in projects. 
However, this method causes damage that can compromise the structure, in addition to using equipment and specialized labor for the removal of testimonies, which makes the process more costly. It is worth highlighting the need for studies and verification of viability by other methods of evaluation, regarding the resistance of the material "in loco", at different ages, without damage to the structural parts to be analyzed. This paper deals with the analysis of the evolution of the axial compression strength of concrete using the rebound hammer, a widely used non-destructive test method that causes little or no damage to the structure. The specimens were made in the Municipality of Curvelo/MG, under the same conditions of trace, cure, humidity and temperature. After its removal, it was transported, in a wet cure, to the Consultare Labcon test laboratory in Belo Horizonte/MG, where the tests of resistance to compression and rebound hammer index were carried out at different ages. The correlation of the axial compression strength of the concrete with the rebound hammer index value was made by generating a calibration curve for the studied concrete. A good correlation index $\left(R^{2}=0.95\right)$ was obtained for the ages analyzed.

Keywords: Non-destructive testing; Rebound hammer; Compressive strength.

\section{Introdução}

A resistência à compressão axial $(\mathrm{RC})$ é uma das propriedades mecânicas mais importantes do concreto, sendo o principal parâmetro que possibilita avaliar a conformidade e comprovar se o material utilizado atende às exigências estabelecidas no projeto. A resistência é influenciada pelas condições como o transporte, lançamento, adensamento e cura do concreto (PALÁCIOS, 2012).

Essa propriedade é de grande importância no âmbito da Construção Civil, para o controle e qualidade de um concreto, além de viabilizar uma durabilidade perfeita ao longo da vida útil de um empreendimento.

Os resultados da RC do concreto são avaliados conforme a idade de rompimento dos corpos de prova (CP), contados a partir da moldagem desses CPs, levando-se em conta a tolerância de tempo prescrita pela NBR 5739 (ABNT, 2018).

De acordo com Palácios (2012), existem vários métodos que permitem a avaliação da resistência in situ das estruturas de concreto. Países como Estados Unidos, Canadá e Alemanha empregam o método dos ensaios não destrutivos (ENDs) na avaliação da condição estrutural. Nesse sentido, vale ressaltar que há um vasto número de pesquisas, na área de avaliação da resistência do concreto, que visam a obtenção de métodos com resultados mais confiáveis.

O ENDs, segundo Evangelista (2002), são aqueles que não causam nenhum ou poucos danos no elemento ensaiado. Esses ensaios não provocam perda na capacidade estrutural da peça. Os ENDs podem ser utilizados em estruturas novas ou antigas, e no caso de estruturas recentes, servem para o monitoramento da evolução da resistência ou mapeamento sobre a qualidade do concreto. Em estruturas mais antigas avaliam sua integridade e capacidade de resistir às solicitações.

Evangelista (2002) detalha que os ensaios não destrutivos podem ser utilizados para mapear fissuras, falhas e danos em meios não homogêneos como o concreto. Eles também são úteis para detectar áreas insalubres ou de concretos suspeitos de estarem, significativamente, abaixo do nível de resistência requerida no projeto ou do nível de durabilidade. O ensaio de esclerometria, juntamente com os outros ENDs, é padronizado nas normas das grandes associações de concreto. Dentre tais normas pode-se citar a American Society for Testing and Materials - ASTM, a Fédération Internationale du Béton - CEB-FIP e o British Standards Institution - BSI.

Os ENDs conquistam cada vez mais espaço no mercado, porém, ao contrário do aço, o concreto é um material heterogêneo de diferentes composições e matérias-primas variadas, o que dificulta a rápida difusão e padronização desta técnica (MALHOTRA; CARINO, 2004).

Este trabalho visa apresentar a evolução da resistência à compressão axial de uma composição específica de concreto ao longo do tempo. Adicionalmente, buscou-se correlacionar a resistência à compressão axial do concreto com o valor do índice esclerométrico através da curva de correlação para uma dosagem específica.

\section{ESTUDO EXPERIMENTAL}


Com o objetivo de traçar uma curva que correlacione a resistência à compressão axial do concreto e o índice esclerométrico, para assim, ser possível a análise da correlação existente entre essas grandezas, o programa experimental englobou três seguimentos. $O$ primeiro foi a moldagem dos $10 \mathrm{CPs}$ : a confecção e dosagem aconteceu na concreteira City Mix Concreto no Município de Curvelo/MG. A segunda etapa foi a de transporte dos CPs em cura úmida, após 24 horas da moldagem, para o laboratório de ensaios Consultare Labcon no Município de Belo Horizonte/MG, onde permaneceram em cura úmida até as idades de ensaio. Foram obtidos os índices esclerométricos, e, logo depois, com os mesmos CPs, foram executados os ensaios de resistência à compressão axial. A terceira etapa foi a tabulação dos resultados dos dois ensaios e a confecção da curva de correlação entre eles, e posterior a análise dos resultados.

\subsection{Materiais}

A moldagem e cura dos CPs são orientados pela NBR 5738 - Moldagem e cura de corpos-de-prova (ABNT, 2015). A amostragem de concreto é orientada pela NBR 16886 - Concreto - Amostragem de concreto fresco (ABNT, 2020). A altura dos moldes cilíndricos utilizados foi igual a $200 \mathrm{~mm}$ e o diâmetro igual a $100 \mathrm{~mm}$. $O$ cimento utilizado foi o CP V-ARI, brita $2(25 \mathrm{~mm})$ e brita $1(12,5 \mathrm{~mm})$ de origem calcária, e areia natural do rio, zona ótima, com módulo de finura 2,59. A relação água/cimento $(a / c)$ utilizada foi igual a 0,52 . Foram moldados 2 CPs cilíndricos para cada idade estudada, de forma a possibilitar a formação de um exemplar para cada idade, e assim, determinar a resistência característica da compressão para cada estudo. $O$ traço unitário utilizado e a quantidade de material para confecção de $17 \mathrm{~L}$ de concreto, em $\mathrm{kg}$, está apresentado na Quadro 1. Essa quantidade foi utilizada para moldar $10 \mathrm{CPs}$, logo, foram avaliados, neste estudo, 5 (cinco) idades distintas do concreto.

Quadro 1 - Traço unitário e quantidade de materiais

\begin{tabular}{|c|c|c|c|c|c|}
\hline Material & Cimento & Brita 2 & Brita 1 & Areia natural & Água \\
\hline unitário & 1 & 1,65 & 1,10 & 1,82 & 0,52 \\
\hline $\mathrm{kg}$ & 13,70 & 22,55 & 15,04 & 24,94 & 6,17 \\
\hline
\end{tabular}

Fonte: Gott (2021) adaptado pelo autor

Os moldes e suas bases, antes de iniciar a moldagem, foram revestidos internamente com uma fina camada de óleo mineral. Foram moldados $10 \mathrm{CPs}$ cilíndricos nas mesmas condições (traço, umidade, temperatura, cura). As amostras foram produzidas com resistência característica à compressão prevista de no mínimo 40 MPa.

\subsection{Ensaio de Esclerometria}

O ensaio esclerométrico utilizando o esclerômetro de reflexão é definido pela norma NBR 7584 - Concreto endurecido - Avaliação da dureza superficial pelo esclerômetro de reflexão - Método de ensaio (ABNT, 2012). Esse ensaio, que mede a dureza superficial do concreto, é um método não destrutivo que fornece dados para a avaliação da qualidade e características do concreto endurecido. De acordo com a NBR 7584 (ABNT, 2012), quanto maior a dureza superficial do concreto endurecido, menor será a parte de energia que se modifica em deformação permanente, então, maior a reflexão ou recuo do objeto que golpea a superfície.

O esclerômetro (Figura 1) é, fundamentalmente, uma massa-martelo que impulsionada por uma mola chocase pela sua haste com ponta em forma de calota esférica com a superfície ensaiada (ABNT, 2012). 

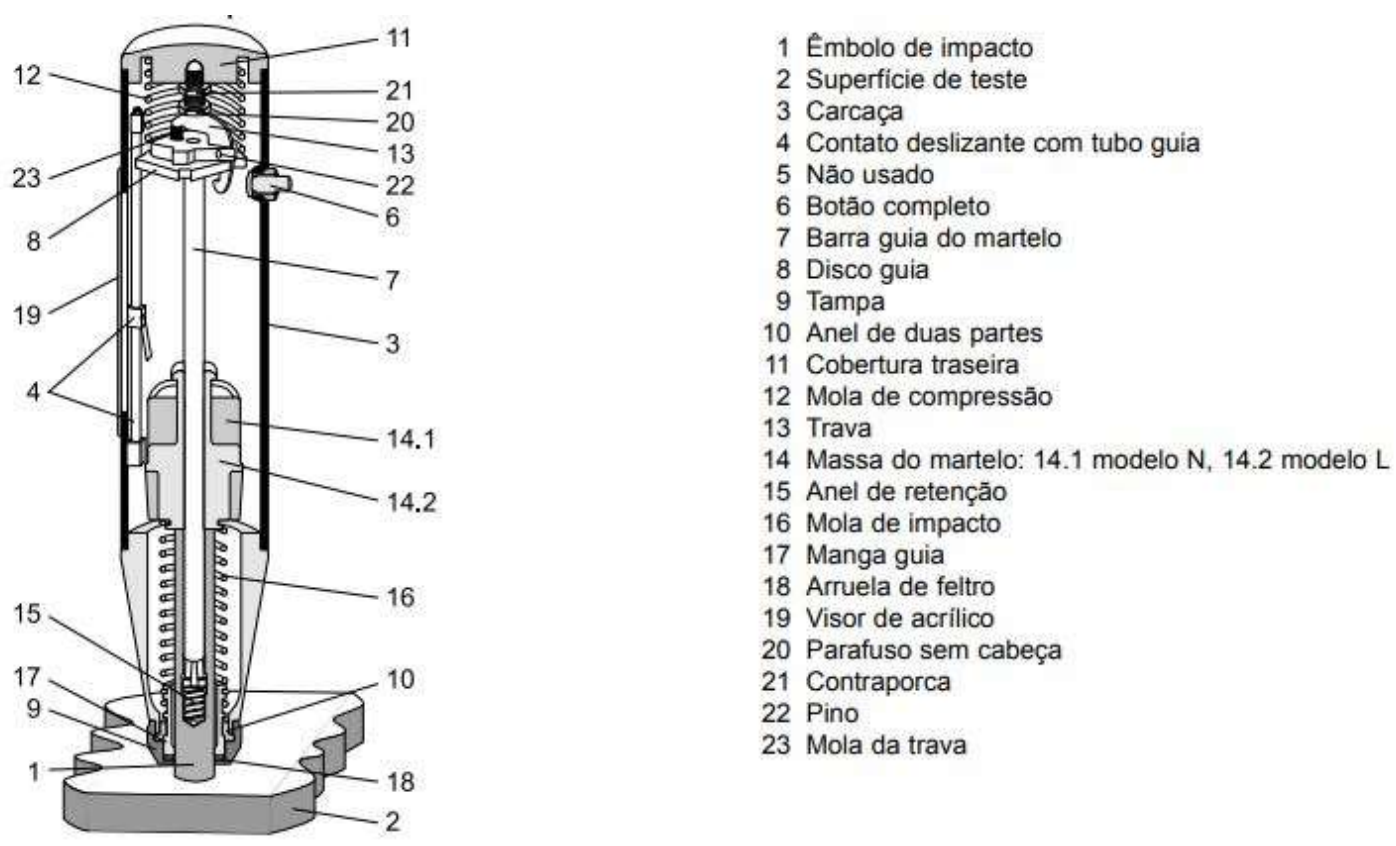

Figura 1 - Seção transversal do matelo para teste do concreto

Fonte: PROCEQ SA (2017)

O método de avaliação da dureza superficial do concreto pelo esclerômetro de reflexão consiste em submeter a superfície do concreto a um impacto de forma padronizada, aplicando uma certa energia e uma certa massa, medindo-se o valor do ricochete, ou seja, o índice esclerométrico. O rebote depende do valor da energia cinética antecedente ao impacto, e quanto desta energia esse impacto absorve. Parte da energia é consumida no atrito mecânico do equipamento, e a outra parte na interação do concreto com a barra de percussão. A energia que é absorvida refere-se à resistência e à rigidez do concreto ( $\mathrm{ACl}, 2004)$.

O equipamento utilizado foi o Esclerômetro para concreto do modelo tipo N, manual, da fabricante Controls com energia de percussão igual a 2,25 N.m. Para a realização do ensaio, de modo a fixar o CP cilíndrico para evitar que, este, se desloque frente ao impacto, foi aplicado, nesse $\mathrm{CP}$, uma força axial de $20 \mathrm{kN}$, por meio da prensa de ensaio. De acordo com a NM 78 (ABNT, 1996), a carga aplicada deve ser equivalente a $15 \%$ da carga de ruptura estimada do CP para evitar interferências com vibrações, ressonância ou dissipação de energia.

O ensaio para determinação do índice esclerométrico foi realizado em todos os CPs cilíndricos nas idades de 3, 5, 16, 25 e 48 dias, sendo 2 CPs para cada idade.

Antes de sua utilização, o esclerômetro foi aferido e ajustado. De acordo com a NBR 7584 (ABNT, 2012), o cilindro foi fixado na prensa, e, com o esclerômetro na posição horizontal, efetuou-se 16 impactos em cada região de ensaio em pontos distintos devidamente distribuídos, como ilustra a Figura 2.

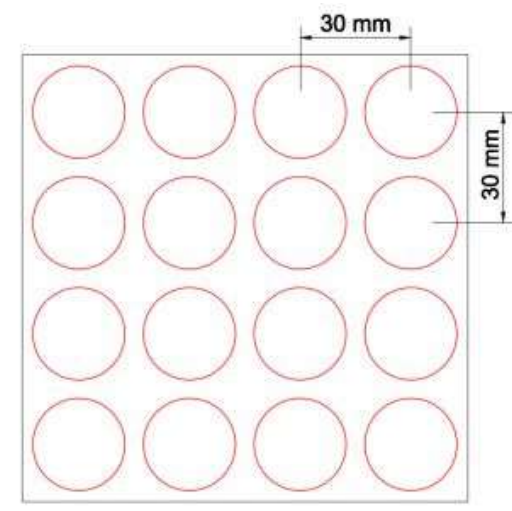

Figura 2 - Área de ensaio

Fonte: NBR 7584 (ABNT, 2012) adaptada pelo autor 
O índice esclerométrico é a leitura da energia de impacto, com unidade de medida adimensional. 0 coeficiente de correção do impacto " $k$ " é obtido por meio da calibração do esclerômetro que é dado pela Equação 1 (NBR 7584, 2012).

$$
k=\frac{n \cdot I E_{\text {nom }}}{\sum_{i=1}^{n} I E_{i}}
$$

Sendo:

k : coeficiente de correção do índice de impacto;

$\mathrm{n}$ : número de impactos na bigorna de aço;

IEnom : índice esclerométrico nominal do aparelho na bigorna (informado pelo fabricante);

IEi : índice esclerométrico obtido em cada impacto do esclerômetro na bigorna.

O índice esclerométrico médio efetivo é obtido pelo produto do coeficiente de correção do índice de impacto "k" pela média dos índices esclerométricos, conforme NBR 7584 (ABNT, 2012). Após realizar os 16 impactos, calcula-se a média aritmética e despreza-se os valores que divergirem em mais de $10 \%$ do valor médio alcançado e recalcula-se a nova média. A cada ensaio é obtido um único índice esclerométrico médio efetivo, conforme a Equação 2, a seguir:

$$
I E_{\propto}=k \cdot I E
$$

Onde:

IE : índice esclerométrico médio;

IE $\alpha$ : índice esclerométrico médio efetivo;

k : coeficiente de correção do índice de impacto.

\subsection{Ensaio de Resistência à compressão axial do concreto}

Os ensaios de resistência à compressão axial do concreto nos CPs foram realizados seguindo as recomendações da NBR 5739 (ABNT, 2018). Utilizou-se, para esse processo, a prensa hidráulica EQUIP - 34 - modelo 2TBO K670 com capacidade de $2000 \mathrm{kN}$ da marca SOLOCAP. O equipamento estava calibrado de acordo com a NBR ISO 7500-1 Materiais metálicos - Calibração e verificação de máquinas de ensaio estático uniaxial- Parte 1: Máquinas de ensaio de tração/ compressão - Calibração e verificação do sistema de medição da força (ABNT, 2016).

Os pratos de compressão e as faces dos CPs foram limpos e secos antes de iniciar o ensaio, e o CP foi centralizado no prato inferior. O carregamento foi aplicado de forma contínua, constante, sem choques e com velocidade de carregamento de $(0,45 \pm 0,15) \mathrm{MPa} / \mathrm{s}$, que foi cessado quando houve uma queda de força (o que indica a ruptura), sendo possível fazer o cálculo da resistência à compressão axial, como prescreve a NBR 5739 (ABNT, 2018) na Equação 3.

$$
f_{c j}=\frac{4 F}{\pi \cdot D^{2}}
$$

Onde,

$f_{c j}$ : resistência à compressão axial, expressa em megapascals (MPa) na idade de j dias;

$\mathrm{F}$ : força máxima alcançada, expressa em newtons $(\mathrm{N})$;

$D$ : diâmetro do corpo de prova, expresso em milímetros $(\mathrm{mm})$.

A relação altura e diâmetro (h/D) foi de 2, não sendo necessário usar um fator de correção adicional.

\section{Resultados e Discussão}

Segundo a NBR 6118 (ABNT, 2014), as estruturas de concreto necessitam ser projetadas e construídas sob as condições ambientais previstas na época e quando utilizadas de acordo com o projeto, conservem sua segurança, estabilidade e aptidão em serviço durante sua vida útil. Portanto, a durabilidade das estruturas está diretamente relacionada com a vida útil prevista em projeto. 
O grande obstáculo é que, em alguns casos, as estruturas em concreto não passam pelo processo de manuntenção preventiva e não atendem a todos os requisitos previstos em norma. Esse descaso pode provocar acidentes, como desabamentos e quedas de estruturas. Tais acontecimentos, por sua vez, podem ser evitados com o controle e monitoramento das edificações, com ENDs (JÚNIOR, 2019).

A utilização do esclerômetro como método de avaliação e monitoramento da resistência ao longo do tempo pode detectar falhas e evitar incidentes. Portanto, é uma ferramenta importante para avaliar a integridade e capacidade das estruturas de resistir às solicitações.

Neste tópico serão apresentados os resultados da esclerometria, bem como o comparativo com os resultados da resistência à compressão axial do concreto para as idades estabelecidas. Foram obtidos dois IE e dois valores de RC para cada idade.

O coeficiente de correção do índice de impacto $\mathrm{k}=0,98$ foi obtido de acordo com o laudo de calibração do esclerômetro, que tem validade até fevereiro de 2022. O Quadro 2 apresenta os valores dos índices esclerométricos médio (IE) e os valores do índice esclerométrico médio efetivo depois da correção do índice de impacto pelo " $k$ " (IE $\alpha$ ) para as idades ensaiadas.

Quadro 2 - Índices esclerométricos do concreto em diferentes idades

\begin{tabular}{|c|c|c|c|c|c|c|c|c|c|c|}
\hline Idade & \multicolumn{2}{|c|}{3 dias } & \multicolumn{2}{c|}{5 dias } & \multicolumn{2}{c|}{16 dias } & \multicolumn{2}{c|}{25 dias } & \multicolumn{2}{c|}{48 dias } \\
\hline IE & 25,53 & 25,60 & 28,19 & 28,33 & 30,81 & 30,60 & 30,88 & 31,25 & 33,63 & 33,19 \\
\hline IE $\alpha$ & 25,02 & 25,09 & 27,62 & 27,77 & 30,20 & 29,99 & 30,26 & 30,63 & 32,95 & 32,52 \\
\hline
\end{tabular}

Fonte: Autores

De acordo com os dados obtidos fica evidente a evolução crescente do IE $\alpha$ com a idade dos CPs. No Quadro 3 estão indicados os valores das resistências à compressão axial dos CPs, de acordo com a Equação 3.

Quadro 3 - Resistência à compressão axial do concreto em diferentes idades

\begin{tabular}{|c|c|c|c|c|c|c|c|c|c|c|}
\hline Idade & \multicolumn{2}{|c|}{3 dias } & \multicolumn{2}{c|}{$\mathbf{5}$ dias } & \multicolumn{2}{c|}{16 dias } & \multicolumn{2}{c|}{25 dias } & \multicolumn{2}{c|}{48 dias } \\
\hline $\begin{array}{c}f_{c j} \\
(\mathrm{MPa})\end{array}$ & 21,39 & 21,11 & 25,96 & 26,45 & 32,32 & 35,59 & 38,18 & 36,55 & 42,04 & 39,99 \\
\hline
\end{tabular}

Fonte: Autores

Como era esperado, tendo em vista as bibliografias consultadas, os maiores valores de resistência à compressão axial foram aqueles registrados para os CPs na idade de 48 dias, sendo de 42,04 MPa e 39,99 $\mathrm{MPa}$, as quais foram 50,9\% e 52,79\% superiores em relação aos 3 dias, respectivamente.

Os resultados obtidos nos dois ensaios são apresentados no gráfico da Figura 3. A equação de correlação entre o índice esclerométrico médio efetivo $(\mathrm{IE \alpha})$ e o valor da resistência à compressão axial do concreto (fcj), para este estudo, é uma função linear. A escolha do grau da equação representativa da curva teve como parâmetro o maior valor para o coeficiente de determinação $\left(R^{2}\right)$. É possível observar uma relação direta entre os valores de resistência à compressão (fcj) e o IE $\alpha$, corroborando com o que é apontado na literatura. 


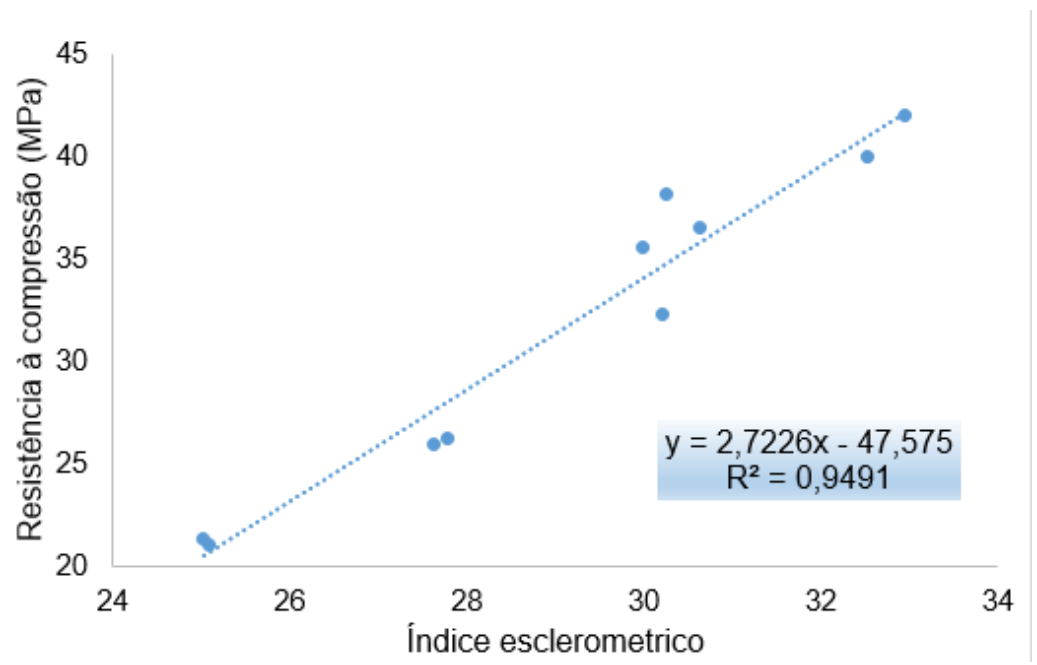

Figura 3 - Correlação do Índice Esclerométrico e da Resistência à compressão axial Fonte: Autores

\section{Conclusões}

A análise estatística da correlação existente entre os ensaios mostrou resultados satisfatórios. Notou-se a existência de uma relação direta e uma boa correlação obtida nos ensaios $\left(R^{2}=0,95\right)$. Essa correlação obtida, tendo em vista seu alto coeficiente de determinação, esclareceu que o ensaio de esclerometria é adequado, principalmente, para a avaliação da uniformidade do concreto empregado na estrutura. Nesse sentido, a pesquisa corroborou a confiabilidade do método e pode servir como parâmetro para ensaios realizados nas mesmas condições deste.

Nessa perpectiva, destaca-se a relevância de pesquisas para disseminar a utilização do ensaio não destrutivo pelo método da esclerometria como opção no âmbito da inspeção e avaliação de estruturas. Todavia, através dos resultados obtidos neste estudo experimental, verificou-se a importância da avaliação de vários fatores, como por exemplo maior número de amostras, relação a/c, tipo de cura, entre outros que são capazes de interferir nos ensaios, a fim de se obter resultados mais confiáveis e representativos.

Em relação ao programa experimental, cabe frisar as limitações referentes à quantidade de CPs e ao número de idades analisadas, que embora tenham atendido aos critérios da norma, foram limitadas para conclusões mais sólidas. Com a conclusão do presente trabalho, pode-se constatar que os objetivos foram alcançados e que os resultados foram satisfatórios, contribuindo para o incentivo de propagação e uso desse método.

\section{Agradecimentos}

Agradecemos ao Centro Federal de Educação Tecnológica de Minas Gerais - CEFET-MG pelo apoio direto e indireto a este trabalho, à empresa City Mix concreto pela doação dos materiais utilizados; à empresa Consultare Labcon, por ter cedido o espaço físico (equipamento e laboratório) para a realização dos experimentos, e por fim, ao LAR - Laboratório de Avaliação e Reabilitação de Ambiente Construído CEFETMG - Campus Curvelo, pelo apoio durante o desenvolvimento deste estudo.

\section{Referências Bibliográficas}

ACI COMMITEE 228. Nondestructive Test Methods for Evaluation of Concrete in Structures, ACl 228.2R98. America, 2004. (Nota técnica).

ASSOCIAÇÃO BRASILEIRA DE NORMAS TÉCNICAS - ABNT. NBR 5739. Concreto - Ensaio de compressão de corpos-de-prova cilíndricos. Rio de Janeiro: ABNT, 2018.

2015. . NBR 5738. Moldagem e cura de corpos-de-prova. Rio de Janeiro: ABNT, 
NBR 7584. Concreto endurecido - Avaliação da dureza superficial pelo esclerômetro de reflexão. Rio de Janeiro: ABNT, 2012.

.NBR NM-ISO 7500-1. Materiais metálicos - Calibração de máquinas de ensaio estático uniaxial - Parte 1: Máquinas de ensaio de tração/compressão - Calibração do sistema de medição da força. ABNT, 2004.

NBR 16886. Concreto - Amostragem de concreto fresco. Rio de Janeiro:

ABNT, 2020.

NM 78. Concreto endurecido - Avaliação da dureza superficial pelo esclerômetro de reflexão. CMN - Comitê MERCOSUL de Normalização, 1996. (Nota técnica).

ASTM C805. Standard Test Method for Rebound Number of Hardened Concrete American Society for Testing and Materials, 2002.

BRITISH STANDARD INSTITUTION. BS 1881: Part 202, 1986, Recommendations for surface hardness testing by rebound hammer", London.

EVANGELISTA, A. C. J. Avaliação da resistência do concreto usando diferentes ensaios não destrutivos. Rio de Janeiro: Tese de Doutorado - Universidade Federal do Rio de Janeiro, 2002.

GOTT, A. P. P.. VARIAÇÃO DA RESISTÊNCIA À COMPRESSÃO AXIAL DO CONCRETO EM IDADE AVANÇADA. 2021. 49 f. TCC (Graduação) (Em andamento).- Curso de Engenharia Civil, Cefet-MG, Curvelo, 2021.

JÚNIOR, R. P. A. Análise da correlação dos ensaios de esclerometria e ultrassom com a resistência à compressão do concreto. TCC (Graduação)- Universidade Federal do Ceará, Centro de Tecnologia, Curso de Engenharia Civil, Fortaleza, 2019.

MALHOTRA, V.; CARINO, N. Handbook on nondestructive testing of concrete. 2 ed. United States of America: CRC Press LLC, 2004.

PALACIOS, M. P. G. Emprego de ensaios não destrutivos e de extração de testemunhos na avaliação da resistência à compressão do concreto. Dissertação (Mestrado em Estruturas e Construção Civil) Faculdade de Tecnologia da Universidade de Brasília. Brasília, 2012.

PROCEQ SA. Instruções operacionais: Martelo de teste de concreto, 2017. Disponível em:<https://www.proceq.com/uploads/tx_proceqproductcms/import_data/files/OrginalSchmidt_Opera ting\%20Instructions_Portuguese_high.pdf> Acesso em: 4 nov. 2020. 